\title{
FUMONISIN PRODUCTION BY FUSARIUM MONILIFORME ISOLATED FROM LEM ASSOCLATED AND NON-LEM ASSTOCIATED CORN
}

\author{
KRISHANTHI ABEYWICKRAMA ${ }^{1^{*}}$ AND GEORGE BEAN ${ }^{2}$ \\ 'Department of Botany, University of Colombo, Colombo. \\ ${ }^{2}$ Department of Botany, University of Maryland, USA.
}

(Received: 03 July 1995; accepted: 06 October 1995)

\begin{abstract}
Qualitative and quantitative analysis of rumonisins produced by $F$. moniliforme isolates associated with an outbreals of leukoencephalomalacia (LEM) in horses were compared to $F$. moniliforme isolates obtained from nonLEM corn. Quantification was by high performance liquid chromatography (HPLC) of o-phthalaldyhyde (OPA) derivatives monitored by fluorescence with confirmation by high performance thin layer chromatography (HPTLC). All four $F$. moniliforme isolates (from LEM and non-LEM corn) produced both fumonisin $\mathrm{B}_{1}\left(\mathrm{FB}_{1}\right)$ and fumonisin $\mathrm{B}_{2}\left(\mathrm{FB}_{2}\right)$. Isolate 33 (from LEM feed corn) produced low levels of $\mathrm{FB}_{1}$ and $\mathrm{FB}_{2}$ compared to isolates from corn not associated with LEM. FB, was the major fumonisio detected in all $F$. moniliforme culture extracts, and its percentage of the total fumonisin was $77 \%-87 \%$ and the remainder was $\mathrm{FB}_{2}$. Results of ow study indicate not only do $F$. moniliforme isolates associated with LEM in horses produce $\mathrm{FB}_{1}$ and $F \mathrm{~B}_{2}$, isolates of F. moniliforme not associated with LEM but present in corn may have even greater potential to produce fumonisins. Current investigation suggests that other fungal metabolite(s) may also be involved in disease development in LEM.
\end{abstract}

Key words: Corn, fumonisins, Fusarium moniliforme, leukoencephalomalacia.

\section{INTRODUCTION}

Field outbreaks of leukoencephalomalacia (LEM) in horses occur sporadically in many countries including South Africa, Argentina, United States and several Asian countries (mainly China). ' Two syndromes associated with LEM in horses are central nervous system (CNS) and hepatotoxicity. The most striking feature of CNS syndrome is liquefactive necrotic lesions in the white matter of the cerebral hemispheres. ${ }^{1}$ The hepatotoxic syndrome is associated with fibrosis, swellings and discoloration of the liver of the affected horses. ${ }^{1}$ Fusarium moniliforme, a common contaminant of corn throughout the world, is believed to be the causative fungus of LEM. ${ }^{1,2}$ An outbreak of this disease occurred in Maryland during the period September 1986 to April 1987 and an estimated 15 horses died in Maryland with classical symptoms of LEM. ${ }^{3}$ Two F. moniliforme types which were later designated as isolates 23 and 33 were identified by Cecile La Grenade from LEM associated feed samples and identity was confirmed by

"Corresponding author 
Paul Nelson, Pennsylvania State University and Robert Goth, USDA, Beltsville. Feed samples implicated in the deaths of these horses were analyzed by Cecile La Grenade for the presence of the mycotoxins moniliformin, fusarin C, zearalenone and some trichothecenes, but none were detected.

Recently two structurally similar compounds called fumonisin $\mathrm{B}_{1}\left(\mathrm{FB}_{1}\right)$ and fumonisin $\mathrm{B}_{2}\left(\mathrm{FB}_{2}\right)$ were isolated from a culture extract of $F$. moniliforme i.e. MRC 826 which when fed to horses caused LEM. ${ }^{1}$ The structure of $\mathrm{FB}_{1}$ has been elucidated by Benzuidenhout et al. (1988) and is a diester of propane-1,2,3 . tricarboxylic acid and a pentahydroxyicosane containing a primary amino group. $\mathrm{FB}_{2}$ is the $\mathrm{C}-10$ deoxy analogue of $\mathrm{FB}_{1} \cdot{ }^{1}$ Fumonisin, produced by $F$. moniliforme, is also reported to be responsible for hydrothorax in swine and carcinogenicity in rats. ${ }^{1,3}$. Feed samples associated with LEM have been surveyed by several researchers for the production of fumonisins, and results indicate that a very wide range of dietary fumonisin concentrations ( $1-122 \mu \mathrm{g} / \mathrm{g}$, are associated with LEM outbreaks in horses. ${ }^{4}$ Reports indicate that higher levels of FB $(1.24-4.0 \mathrm{mg} / \mathrm{kg} /$ day) are needed to experimentally reproduce LEM in equids by intravenous injection compared to the relatively low $\mathrm{FB}$, intake by horses showing natural outbreak of LEM $(0.6-2.1 \mathrm{mg} / \mathrm{kg} / \mathrm{day}){ }^{4}$ Most of the earlier laboratory and feeding studies with LEM in horses have been done with the South African isolate MRC 826. We report on the fumonisin production by $F$. moniliforme isolates associated with an outbreak of LEM in horses in Maryland between 1986 - 1987 in comparison to $F$. monoliforme isolates obtained from feed corn not associated with LEM. Non-LEM or relatively "clean" corn was included in this study because previous survey of fungi in corn kernels from several countries in Maryland indicated that corn kernels are typically contaminated with the fungus $F$. moniliforme ${ }^{5}$ Frequency of occurrence of the above fungus ranged from $44.61 \% .^{5}$ Further the contamination levels of this fungus before and during storage changes very little. The current investigation was also done to determine whether furnonisins alone are responsible for symptom development in LEM or other fungal metabolites are also involved in thirs durseaseịn equiờs. In corn and ot̂her stored grains łungi are rarély ever found alone on the same grain or kernel.

\section{METHODS AND MATERIALS}

Isolation of $F$. moniliforme: Three samples of field corn (not-associated with LEM) were obtained from the Cooperative Extension Service, College Park, Maryland, USA. The 3 samples were mixed thoroughly. One hundred randomly selected kernels of the 3 mixed non-LEM samples were surface disinfected in a $10 \%$ clorox solution containing $0.525 \% \mathrm{NaOCl}$ and plated on salt $(7.5 \% \mathrm{NaCl})$ PDA (potato dextrose agar) (Difco Laboratories, Detrait, MI) ${ }^{3,6}$ After $7 \mathrm{~d}$ at $20^{\circ} \mathrm{C}$ 2 isolates of $F$. moniliforme (isolates 1 and 2 ) were identified. ${ }^{7,8}$ Two additional isolates of the same fungus designated 23 and 33 were isolated by Cecile Granade originally from corn associated with an outbreak of LEM in Maryland in 1986- 
1987. Identity of $F$. moniliforme isolates were confirmed by Paul Nelson, Pennsylvania State University and Robert Goth, USDA, Beltsville.

Growth of $F$. moniliforme isolates on solid media for fumonisin production: $50 \mathrm{~g}$ of cracked corn and $11 \mathrm{ml}$ distilled water were added to a $500 \mathrm{ml}$ Erlenmeyer flask and autoclaved for $1 \mathrm{~h}$ at $121^{\circ} \mathrm{C}$ and $16 \mathrm{lb}$ pressure. After cooling, $11 \mathrm{ml}$ of sterile distilled water was added to each flask which were then inoculated with a spore suspension ( 106 conidia/ml) of each of four $F$. moniliforme isolates $(1,2,23$ and. 33) and incubated for 4 weeks at $25^{\circ} \mathrm{C} .9^{9}$ After incubation, the corn was dried for $5 \mathrm{~d}$ at room temperature and subjected to fumonisin analysis.

Extraction of fumonisin: $F$. moniliforme inoculated corn cultures (10 g) were ground in a Waring blender, mixed with $50 \mathrm{ml}$ acetonitrile/water $(50 / 50, \mathrm{v} / \mathrm{v})$, shaken for $30 \mathrm{~min}$ on a wrist action shaker, and filtered through filter paper (qualitative fluted, grade: $315,24 \mathrm{~cm}$ diameter). $2 \mathrm{ml}$ of filtered extract was transferred to a $15 \mathrm{ml}$ vial and $5 \mathrm{ml}$ of distilled water was added. The extract was loaded onto a C-18 cleanup cartridge (Sep-pak, Waters Associates, Milford, $\mathrm{MA})$, rinsed with $2 \mathrm{ml}$ of water followed by $2 \mathrm{ml}$ acetonitrile/water $(20 / 80, \mathrm{v} / \mathrm{v})$. The fumonisins present were eluted with $2 \mathrm{ml}$ acetonitrile/water $(70 / 30, \mathrm{v} / \mathrm{v}), 6,20$

Analysis offumonisin by HPTLC: The previous Sep-pak cleaned culture extracts were spotted on HPTLC (High Performance Thin layer Chromatography silica gel 60 plates; $10 \times 10 \mathrm{~cm}$; E. Merck AG, Darmstadt, Germany) along with fumonisin $\mathrm{B}_{1}\left(\mathrm{FB}_{1}\right)$ and fumonisin $\mathrm{B}_{2}\left(\mathrm{FB}_{2}\right)$ standards purchased from CSIR, Pretoria, South Africa. After development in 60/30/10 (chloroform/methanol acetic acid, $\mathrm{v} / \mathrm{v} / \mathrm{v})$ the plates were air dried, sprayed with $\mathrm{p}$-anisaldehyde $(0.5 \%$ in methanol/sulfuric acid/acetic acid, 90/5/5, v/v/v) and heated at $100^{\circ} \mathrm{C}$ for $5 \mathrm{~min}$. The levels of fumonisin in spots corresponding to fumonisin standards were estimated as described in the literature. 6.10

Identification of fumonisin using HPLC: A modified method of Ross et al. (1991) and Shephard et al. (1990) was used for HPLC (High Performance Liquid Chromatography) analysis. ${ }^{210}$ Culture extracts obtained after Sep-pak cleanup were reacted with o - phthalaldehyde (OPA) to produce fluorescent derivatives of $\mathrm{FB}_{1}$ and $\mathrm{FB}_{2}$. The OPA reagent was prepared by dissolving $40 \mathrm{mg}$ OPA in $1 \mathrm{ml}$ acetonitrile and $5 \mathrm{ml} 0.1 \mathrm{M}$ borate buffer $(\mathrm{pH} \mathrm{9.0)}$ ) and $50 \mu \mathrm{l}$ of mercaptoethanol. Due to the instability of the OPA derivative, $50 \mu \mathrm{l}$ of culture extracts were mixed with $50 \mu$ l of OPA reagent 5 min prior to injection. The derivatised samples plus standards were analyzed by a reverse-phase, linear HPLC system (Gilson, model 302 ) equipped with a fluorichrom (Varian, Walnut Creek, CA) with $335 \mathrm{~nm}$ and $440 \mathrm{~nm}$ excitation and emission filters. The analytical column ( $250 \mathrm{~mm} \times 4.6 \mathrm{~mm}$ ) was packed with octadecyl silane (ODS), 3 micron reverse - phase (C-18) material. Reverse - phase (30 mm x $4.6 \mathrm{~mm}$ ) cleanup precolumn packed with 3 micron adsorbent material was used to protect the analytical column. Eluents 
were acetonitrile/0.1M sodium dihydrogen phosphate (40/60) (system A) and acetonitrile/0.1M sodium dihydrogen phosphate (60/40) (system B) adjusted to $\mathrm{pH} 3.3$ with phosphoric acid. Solvent system A was used for 10 min and then solvent system $B$ at a flow rate of $0.5 \mathrm{ml} / \mathrm{min}$. Peak retention times were noted, and compared to the peak retention times of $\mathrm{FB}_{1}$ and $\mathrm{FB}_{2}$ standards.6,10 For further identity verification, $\mathrm{FB}_{1}$ and $\mathrm{FB}_{2}$ standards were mixed with culture extracts which were then derivatised with OPA and after injection $(20 \mu 1)$ the number of peaks and retention times were noted.

Quantitation of fumonisin using HPLC:

(a) Preparation of $\mathrm{FB}_{1}$ and $\mathrm{FB}_{2}$ standards: Solutions containing $0.8-2.4 \mu \mathrm{g} \mathrm{FB}$ standard in OPA were prepared as well as OPA derivative solutions containing $0.2-2.0 \mu \mathrm{gBB}_{2}$. These solutions were freshly made before each injection. Conditions for HPLC analysis were the same as previously described, $20 \mu 1$ injections of the fumonisin- OPA derivatives were made and the retention times and peak areas were recorded. Each concentration was injected 3 times and mean peak area values were calculated. ${ }^{6}$

(b) Analysis of $F$. moniliforme culture extracts: Culture extracts of $F$. moniliforme were mixed with OPA at $1: 1(\mathrm{v} / \mathrm{v})$ ratio and the presence and levels of fumonisins were determined as previously described. Each culture extract was injected 3 times. A graph of mean peak areas and concentrations of $\mathrm{FB}_{1}$ and $\mathrm{FB}_{2}$ standards were plotted and a best fit line was drawn. Using a linear regression model, the concentrations of $\mathrm{FB}_{1}$ and $\mathrm{FB}_{2}$ in the culture extracts were calculated. ${ }^{6}$

\section{RESULTS}

Even though corn kernels were plated on salt PDA to facilitate slower growing fungi, the predominant fungal species isolated from two non-LEM corn samples were sclerotial and non-sclerotial forming Aspergillus flavus at frequencies 18 and $30 \%$ and 68 and $92 \%$, respectively. Two $F$. moniliforme types were also isolated which were later designated as isolate 1 and 2. Based on HPTLC analysis, culture extracts from all $4 F$. moniliforme isolates $(1,2,23,33)$ contained $\mathrm{FB}_{1}$ which appeared as blue-purple spots at Rf's between 0.35-0.88. Gelderblom et al. (1988) found similar Rf values for fumonisin present in culture extracts of the South African F. moniliforme isolate. ${ }^{11}$ The amounts of $\mathrm{FB}_{1}$ produced by $F$. moniliforme isolates 1 and 2 (not associated with LEM) were similar (500 \pm 77 and $520 \pm 79 \mu \mathrm{g} / \mathrm{g}$ ), while culture extracts from the LEM com isolate, i.e. 33, contained approximately four times less $\mathrm{FB}_{1}(110 \pm 98 \mu \mathrm{g} / \mathrm{g})$ than was produced by the isolates not associated with LEM (Table 1). The level of FB produced by the other LEM isolate, i.e. 23, was similar in amounts to the non-LEM isolates $(435 \pm 188 \mu \mathrm{g} / \mathrm{g}) . \mathrm{FB}_{2}$ could not be detected in any of the culture extracts using HPTLC. However, $\mathrm{FB}_{1}$ and $\mathrm{FB}_{2}$ were detected in the 4 culture extracts using HPLC. Single peaks were obtained for the OPA derivatized $F B_{1}$ and $F B_{2}$ standards as well as fumonisins present in the culture extracts at a retention 
time of 13-15 and 18-20 min, respectively. Culture extracts of isolate 1 contained the highest level of $\mathrm{FB}_{1}(680-760 \mu \mathrm{g} / \mathrm{g})$, while culture extracts of isolates 2 and 23 contained similar levels of $\mathrm{FB}_{1}(560-600 \mu \mathrm{g} / \mathrm{g})($ Table 2$)$. Isolate 33 contained the least amount of $\mathrm{FB}_{1}(140-150 \mu \mathrm{g} / \mathrm{g})$. Isolate 33 also produced the lowest level of $\mathrm{FB}_{2}(40 \mu \mathrm{g} / \mathrm{g}$ corn $)$ compared to $90-170 \mu \mathrm{g} / \mathrm{g}$ of corn produced by the other isolates. Further, standard deviations of mean fumonisin levels obtained by HPLC was low compared to values obtained by HPTLC suggesting that HPLCfluorescence detection is a more reliable and a precise technique of quantifying fumonisins. In all cases, $\mathrm{FB}_{1}$ was the major fumonisin detected, and the ratios $\mathrm{FB}_{1}: \mathrm{FB}_{2}$ were approximately $80: 20 .^{1}$ Thiel et al. (1991) reported $\mathrm{FB}_{1}: \mathrm{FB}_{2}$ ratios of $80: 20$ as well as $96: 4$ in $F$. moniliforme inoculated corn culture extracts. ${ }^{1}$

Table 1: FB $_{1}$ levels in corn inoculated with $F$. moniliforme analyzed by HPTLC.

\begin{tabular}{cc}
\hline Isolate $^{\mathrm{a}}$ & $\begin{array}{c}\text { Mean } \mathrm{FB}_{1}^{\mathrm{b}} \pm \text { standard deviation } \\
(\mu \mathrm{g} / \mathrm{g} \text { of corn })\end{array}$ \\
\hline 1 & $500 \pm 77$ \\
2 & $520 \pm 79$ \\
23 & $435 \pm 188$ \\
33 & $110 \pm 98$ \\
\hline
\end{tabular}

- Isolates 1 \& 2 from clean corn and isolates 23 \& 33 from LEM corn.

b. Each number is the mean of 6 replicates.

Table 2: $\quad \mathrm{FB}_{1}$ and $\mathrm{FB}_{2}$ levels in corn inoculated with $F$. moniliforme analyzed by HPLC.

\begin{tabular}{|c|c|c|c|}
\hline \multirow[b]{2}{*}{ Isolate } & \multicolumn{3}{|c|}{ Fumonisin $(\mu \mathrm{g} / \mathrm{g} \text { of } \operatorname{corn})^{\mathrm{b}}$} \\
\hline & $\mathrm{FB}_{1} \pm$ standard deviation & $\mathrm{FB}_{1} \%$ & $\mathrm{FB}_{2}$ \\
\hline \multirow[t]{2}{*}{1} & $680 \pm 40$ & 85 & 120 \\
\hline & $760 \pm 50$ & 87 & 110 \\
\hline \multirow[t]{2}{*}{2} & $600 \pm 32$ & 79 & 160 \\
\hline & $580 \pm 38$ & 77 & 170 \\
\hline \multirow[t]{2}{*}{23} & $580 \pm 42$ & 84 & 110 \\
\hline & $560 \pm 30$ & 86 & 90 \\
\hline \multirow[t]{2}{*}{33} & $140 \pm 10$ & 78 & 40 \\
\hline & $150 \pm 09$ & 79 & 40 \\
\hline
\end{tabular}

- Isolates 1 \& 2 from clean corn and isolates 23 \& 33 from LEM corn.

- Each number is the mean of 3 replicates. 


\section{DISCUSSION}

Fumonisins have been suggested and experimentally shown to be responsible in causing LEM disease in equids.,10,12 Results of our study indicate that not only do $F$. moniliforme isolates associated with $L E M$ in horses produce $F B_{1}$ and $F B_{2}$, but isolates of $F$. moniliforme from corn not directly associated with LEM are also able to produce fumonisins at similar or even higher concentrations. The natural occurrence of $\mathrm{FB}_{1}$ and $\mathrm{FB}_{2}$ in substrates and agricultural commodities contaminated with different isolates of $F$. moniliforme has been reported. ${ }^{32}$ The same researcher compared fumonisin production amongst different species of Fusarium, and found that in addition to $F$. moniliforme, F. proliferatum and $F$. nygamai were also able to produce $\mathrm{FB}_{1}$ and $\mathrm{FB}_{2}$ at levels ranging from $20-660$ $\mu \mathrm{g} / \mathrm{g}$ and $65-450 \mu \mathrm{g} / \mathrm{g}$, respectively. ${ }^{12}$ Further investigations conducted using the same four $F$. moniliforme isolate culture extracts that were used in the current study, and fumonisin standards, revealed that $\mathrm{FB}_{1}$ and $\mathrm{FB}_{2}$ present in extracts are responsible for causing inhibition in cell proliferation and cytotoxicity to two mammalian cell lines: baby hamster kidney (BHK-21) and human epidermoid (HEP-2). ${ }^{6}$ Radiolabeled experiments revealed that $\mathrm{FB}_{1}$ is involved in the disruption of RNA synthesis, and reduced RNA levels in BHK-21 cells at 24 and $48 \mathrm{~h}$ incubation times. ${ }^{6}$ However, research conducted by three groups of workers using feeds associated with LEM in equids, indicated that low levels of both $\mathrm{FB}_{1}$ $(1-122 \mu \mathrm{g} / \mathrm{g})$ and $\mathrm{FB}_{2}(0.1-1.23 \mu \mathrm{g} / \mathrm{g})$ occur in corn whereas in our study by inoculating corn with $F$. moniliforme isolates and providing optimum conditions for growth, we were able to facilitate production of much higher levels of $F B_{1}$ $(140-760 \mu \mathrm{g} / \mathrm{g})$ and $\mathrm{FB}_{2}(40-170 \mu \mathrm{\mu g} / \mathrm{g}){ }^{2.4 .12} \mathrm{FB}_{1}$ and $\mathrm{FB}_{2}$ levels as much as 7100 and $3000 \mu \mathrm{g} / \mathrm{g}$ has been reported. ${ }^{12}$ Current study and other related investigations indicate that $F$. moniliforme is always producing fumonisins on corn. The question raised here was what factors trigger sporadic and erratic occurrence of LEM in equids? Can relatively low levels of fumonisins naturally present in feed samples cause LEM disease in equids? Are fungi other than $F$. moniliforme and other metabolites also involved? Both Aspergillus fumigatus and $F$. moniliforme have been isolated from LEM corn samples and 'relatively clean' corn during this survey. There are published reports on the ability of the above two fungi to occur/ grow simultaneously in/on corn. ${ }^{13}$ Aspergillus flavus strains can produce aflatoxins (mainly $\mathrm{B}_{1}$ and $\mathrm{B}_{2}$ ) on stored corn under favourable environmental conditions. ${ }^{13}$ The presence of both fungi in corn simultaneously raises the question as to whether aflatoxins are also involved in LEM disease in equids. Vesonder et $\alpha l$. (1991) reported that aflatoxins in corn feed were compatible with the diagnosis of equine aflatoxicoses a disease which mainly affects the liver of horses. ${ }^{14}$ Their studies suggest that aflatoxins occurring natural in corn could influence the response of equids to fumonisins which are produced by $F$. moniliforme and $F$. proliferatum on the same substrate. Recently it was reported that Aspergillus fumigatus produces a metabolite known as "sphingofungin" which has broad spectrum antifungal activity. ${ }^{15,16}$ Sphingofungins which have 
structural similarities to sphingolipid related long-chain bases, like fumonisins, have been shown to cause a decrease in sphingolipid biosynthesis in Saccharomyces cerevisiae, by inhibiting palmitoyltransferase, the first enzyme in the sphingolipid catabolism pathway. ${ }^{15,16}$ Since Norred et al.(1992) ${ }^{17}$ have demonstrated the ability of fumonisins in causing a decrease in sphingolipid biosynthesis in mammalian cells by inhibiting ceramide synthase, the second enzyme in the sphingolipid pathway, clinical and pathological symptoms in equids with LEM disease could thus be due to the combined effect of both fumonisins and sphingofungins. Future experiments should be focussed on screening for the presence of both $F$. moniliforme, $F$. proliferatum and Aspergillus species in LEM-associated corn and other feeds, and their potential of producing both fumonisins and sphingofungins should be evaluated. Involvement of fumonisins and various other fungal metabolites and their synergistic effect in symptom development in LEM disease is yet to be evaluated.

\section{Acknowledgement}

Assistance with the HPLC analysis by Jarvis and Shehgiun Wong, Department of Chemistry, University of Maryland is gratefully acknowledged. We wish to thank Ronald Plattner of USDA, Peoria, Illinois for information on fumonisin production by Fusarium moniliforme, and Paul Nelson, Robert Goth and Cecile La Grenade for their assistance in identifying $F$. moniliforme. We thank Lasantha Abeywickrama for assistance in regression analysis and Cindy Wong for her help during the project.

\section{References}

1. Thiel P.G., Shephard G.S., Sydenham E.W., Marasas W.F.O., Nelson P.E. \& Wilson T.M. (1991). Levels of fumonisins $B_{1}$ and $B_{2}$ in feeds associated with confirmed cases of equine leukoencephalomalacia. Journal of Agricultural and FoodChemistry 39: 109-111.

2. Shephard G.S., Sydenham E.W., Thiel P.G. \& Gelderblom W.C.A. (1990). Quantitative determination of fumonisin $B_{1}$ and $B_{2}$ by high-performance liquid chromatography with fluorescence detection. Journal of Liquid Chromatography 13: 2077-2087.

3. La Grenade Cecile. (1990). Studies on Fusarium moniliforme causal agent of equine leucoencephalomalacia. Ph. D. Thesis. University of Maryland, College Park, Maryland, USA.

4. Thiel P.G., Marasas W.F.O., Sydenham E.W., Shephard G.S. \& Gelderblom W.C.A. (1992). The implications of naturally occurring levels of fumonisins in corn for human and animal health. Mycopathologia 117: 3-9. 
5. Trofa S. \& Bean G.A. (1987). The level of toxigenic fungi in 1985 Maryland corn before and during storage. Biodeterioration Research 1: 119-126.

6. Abeywickrama K. (1993). The involvement of Fusarium moniliforme and fumonisins in Leucoencephalomalacia and Type A spiking mortality disorder. Ph.D. Thesis. University of Maryland, College Park, USA.

7. Marasas W.F.O., Nelson P.E. \& Toussoun T.A. (1983). Toxigenic Fusarium species: identity and mycotoxicology. pp. 1-193. Pennsylvania State University Press, Pennsylvania, USA.

8. Nelson P.E., Toussoun T.A. \& Marasas W.F.O. (1983). Fusarium species: an illustrated manual for identification. pp. 1-205. Pennsylvania State University Press, Pennsylvania, USA.

9. Plattner R.D., Weisler D., Shackleford D.D., Peterson R. \& Lowell R.G. (1992). A new fumonisin from solid cultures of Fusarium moniliforme. Mycopathologia 117: $23-28$.

10. Ross P.F., Rice L.G., Plattrier R.D., Osweiler G.D., Wilson T.M., Owens D.L., Nelson A.A. \& Richard J.L. (1991). Concentrations of fumonisin $B_{3}$ in feeds associated with animal health problems. Mycopathologia 114:129-135.

11. Gelderblom W.C.A., Jaskiewicz K., Marasas W.F.O., Thiel P.G., Horak R.M., Vleggaar R. \& Kriek N.P.J. (1988). Fumonisins - Novel mycotoxins with cancer-promoting activity produced by Fusarium moniliforme. Applied Microbiology 54: 1806-1811.

12. Thiel P.G., Marasas W.F.O., Sydenham E.W., Shephard G.S., Gelderblom W.C.A. \& Nienwenhuis J.J. (1991). Survey of fumonisin production by Fusarium species. Applied and Environmental Microbiology 57:1089-1093.

13. Wicklow D.T. (1983). Taxonomic features and ecological significance of sclerotia in aflatoxin and Aspergillus flavus in corn. pp. 20-60. Department of Research Information, Alabama Agricultural Experiment Station, Auburn University, Alabama, USA.

14. Vesonder R., Haliburton J., Stubblefield R., Gilmore W. \& Peterson S. (1991). Aspergillus flavus and aflatoxins $\mathrm{B}_{1}, \mathrm{~B}_{2}$ and $\mathrm{M}_{1}$ in corn associated with equine death. Archives of Environmental Contamination and Toxicology 20: 151-153. 
15. VanMiddlesworth F., Giacobbe R., Lopez M., Garrity G., Bland J.A., Bartizal K., Fromtling R.A., Polishook J., Zweerink M., Edison A.M., Rodzilsky W., Wilson K.E. \& Monaghan R.L. (1992). Sphingofungins A, B,C, D: a new family of antifungal agents - fermentation, isolation, and biological activity. Journal of Antibiotics 45: 861-867.

16. Zweerink M.M., Edison A.M., Wells G.B., Pinto W. \& Lester R. (1992). Characterization of a novel, potent, and specific inhibitor of serine palmitoyltransferase. Journal of Biological Chemistry 267: 25032-25038.

17. Norred W.P., Wang E., Yoo Hwansoo., Riley R.T. \& Merrill Jr. A.H. (1992). In vitro toxicology of fumonisins and the mechanistic implications. Mycopathologia 117: 73-78. 